\title{
Algunos aspectos sobre el desarrollo profesional de la medicina evaluadora de incapacidades laborales
}

\section{Some aspects of the professional development of Evaluative Medicine of incapacity for work}

\author{
Luis Sánchez-Galán ${ }^{1}$ \\ Pilar Baides-Gonzalvo ${ }^{1}$ \\ Raúl Regal-Ramos ${ }^{1}$ \\ Gema Herreros-Portolés ${ }^{1}$
}

${ }^{1}$ Unidad Médica del Equipo de Valoración de Incapacidades. Dirección Provincial del Instituto Nacional de la Seguridad Social. Madrid. España.

\section{Correspondencia}

Luis Sánchez Galán

luis.sanchezgalan@gmail.com

Recibido: 22.03.2021

Aceptado: 26.03.2021

Publicado: 31.03 .2021

\section{Contribuciones de autoría}

Las autoras y los autores de este trabajo han contribuido por igual.

Financiación

Sin financiación.

\section{Conflicto de intereses}

Se señala la no existencia de conflicto de intereses para los autores del presente artículo. 


\section{Resumen}

La medicina evaluadora de incapacidades laborales es aquella rama u orientación de la medicina evaluadora que tiene por objetivo valorar la repercusión funcional del cuadro clínico de un trabajador, a efectos de que se determine la existencia o no de algún tipo y grado de incapacidad laboral. Es, por tanto, una aplicación práctica de la medicina que queda fuera del ámbito asistencial y que es ejercida, entre otros, por los médicos inspectores adscritos a la Seguridad Social en España. En el momento presente, los autores consideran necesario el desarrollo e implantación en España de unos estudios específicos que cualifiquen para la práctica de esta rama de la medicina, que pueden encajar como una modalidad de área de capacitación específica para diferentes especialidades médicas, buscando así dotar del mayor rigor científico en las evaluaciones médicas. Igualmente consideran necesaria, en el ámbito médico de la Seguridad Social, el desarrollo de oportunidades de ascenso y expectativas de progreso profesional conforme a los principios de igualdad, mérito y capacidad, lo que implica un sistema de carrera profesional.

Palabras clave: medicina evaluadora; incapacidad laboral; carrera profesional; área de capacitación específica.

\section{Abstract}

Evaluative Medicine of incapacity for work is a branch or orientation of Medicine that aims to assess the functional repercussions of a worker's clinical picture, for the purpose of determining whether there is some type and degree of work disability. It is, therefore, a practical application of Medicine that is outside the scope of health care and is carried out, among others, by the medical officers assigned to the Social Security (National Health Service) in Spain. At the present time, the authors consider it necessary to develop and implement specific studies in Spain to qualify for the practice of this branch of Medicine, which could fit in as a specific training area for different medical specialties, thus seeking to provide greater scientific rigor in medical evaluations. They also deem it necessary, in the medical field of Social Security, to develop opportunities for promotion and expectations of professional progress in accordance with the principles of equality, merit and ability, which are implied in a professional career system

Keywords: Evaluative Medicine; work incapacity; professional career; specific training area. 


\section{Introducción}

Ante la pregunta de ¿qué es la medicina evaluadora? se ha respondido que es aquella actividad médica específica que estudia las diferentes enfermedades o trastornos que padece una persona a efectos de determinar su magnitud y su repercusión sobre diferentes aspectos de la vida de esa persona o de la relación de esta con el entorno social ${ }^{(1,2)}$.

La medicina evaluadora de incapacidades laborales es aquella rama u orientación de la medicina evaluadora que tiene por objetivo valorar la repercusión funcional del cuadro clínico de un trabajador a efectos de que se determine la existencia o no de algún tipo y grado de incapacidad laboral ${ }^{(2)}$. Es por tanto una aplicación práctica de la medicina que queda fuera del ámbito asistencial.

El estudio y la consideración de las limitaciones orgánicas y funcionales en cada trabajador en referencia a los requerimientos físicos de las diferentes ocupaciones laborales y profesión habitual han sido objeto de un conocimiento médico específico y aplicado por la medicina evaluadora.

La metodología que aplica la medicina evaluadora se basa fundamentalmente en dos grupos de métodos: 1. Métodos negativos: La valoración se basa en una serie de listados que indican enfermedades y características de las mismas que impiden la realización de alguna profesión u oficio (requerimiento de permisos o licencias). 2. Métodos positivos: La valoración consiste en medir la capacidad funcional de la persona. Básicamente los métodos positivos se pueden resumir según tres metodologías de trabajo positivas: Las primeras son descriptivas: se basan en la descripción y valoración subjetiva del médico evaluador, sobre las lesiones presentes. Las segundas se basan en baremos: identifican determinadas lesiones o enfermedades con una cantidad indemnizatoria o con puntuación (puntos o porcentaje). En tercer lugar, la metodología empírica, muy utilizada en medicina evaluadora, aplica una determinada sistemática de exploración basada en protocolos y valoración individualizada de cada caso ${ }^{(1,2)}$.

La afectación del estado de salud del trabajador y sus consecuencias sobre la capacidad de desarrollar su trabajo puede llegar a ocasionar una interrupción de su actividad laboral acarreando una situación de necesidad por la falta de ingresos económicos que ello supone.

\section{Situación actual}

La Constitución Española de 1978 establece en su artículo 41 que los poderes públicos mantendrán un régimen público de Seguridad Social para todos los ciudadanos que garantice la asistencia y prestaciones sociales suficientes ante situaciones de necesidad ${ }^{(3)}$.

La Ley General de la Seguridad Social ${ }^{(4)}$ fundamenta el sistema en los principios de universalidad, unidad, solidaridad e igualdad, y expone la acción protectora del sistema de la Seguridad Social, que comprenderá:

a) La asistencia sanitaria en los casos de maternidad, de enfermedad común o profesional y de accidente, sea o no de trabajo.

b) La recuperación profesional, cuya procedencia se aprecie en cualquiera de los casos que se mencionan en la letra anterior.

c) Las prestaciones económicas en las situaciones de incapacidad temporal, nacimiento y cuidado de menor, riesgo durante el embarazo, riesgo durante la lactancia natural, ejercicio corresponsable del cuidado del lactante, cuidado de menores afectados por cáncer u otra enfermedad grave, incapacidad permanente contributiva e invalidez no contributiva, jubilación, en sus modalidades contributiva y no contributiva, desempleo, en sus niveles contributivo y asistencial, protección por cese de actividad, pensión de viudedad, prestación temporal de viudedad, pensión de orfandad, prestación de orfandad, pensión en favor de familiares, subsidio en favor de familiares, auxilio por defunción, indemnización en caso de muerte por accidente de trabajo o enfermedad profesional, ingreso mínimo vital, así como las que se otorguen en las contingencias y situaciones especiales que reglamentariamente se determinen por real decreto a propuesta del titular del Ministerio competente. 
d) Las prestaciones familiares de la Seguridad Social en sus modalidades contributiva y no contributiva.

e) Las prestaciones de servicios sociales que puedan establecerse en materia de formación y rehabilitación de personas con discapacidad y de asistencia a las personas mayores, así como en aquellas otras materias en que se considere conveniente.

En este marco de prestaciones de la seguridad social, algunas de ellas relacionadas con la salud y la enfermedad, la medicina evaluadora se ha ido desarrollando como una necesidad de perfeccionamiento profesional en la continua mejora de la aplicación del estado del bienestar.

Desde 1995, año en el que el Real Decreto 1300/1995 de 21 de julio(5) establece la creación de los Equipos de Valoración de Incapacidades (EVI), uno de los integrantes de estos equipos es un facultativo médico perteneciente al Instituto Nacional de la Seguridad Social (INSS). Para ello se adscriben al Instituto Nacional de la Seguridad Social, como médicos evaluadores, los facultativos de la Escala de Médicos Inspectores del Cuerpo de Inspección Sanitaria de la Administración de la Seguridad Social. Entre las funciones de estos EVI se encuentra la de evaluar, calificar y revisar la incapacidad y reconocer el derecho a las prestaciones económicas contributivas de la Seguridad Social por invalidez permanente en sus distintos grados, así como determinar las contingencias causantes de la misma.

Aproximadamente 650 facultativos constituyen hoy la plantilla de médicos inspectores adscritos al INSS, integrados en la estructura de la Dirección General del Instituto Nacional de la Seguridad Social a través de una Subdirección General, así como en las diferentes Unidades Médicas de las Direcciones Provinciales del INSS ${ }^{(6)}$. Con el paso del tiempo se vienen planteado necesidades formativas y estructurales con el objetivo de conseguir el mejor rigor científico técnico posible en el cumplimiento de la función de estas unidades ${ }^{(7,8)}$.

\section{Algunos aspectos para el desarrollo profesional de la medi- cina evaluadora}

Entre estas necesidades, a juicio de los autores de este trabajo, se continúan evidenciando dos, como clave de bóveda, para el progreso profesional de esta área de la medicina:

En primer lugar, el desarrollo de la carrera profesional en el ámbito de la medicina evaluadora para los médicos evaluadores de la seguridad social.

El amparo normativo de esta carrera se puede encontrar en la Ley del Estatuto Básico del Empleado Público de 2015, que en su artículo 16 recoge que los funcionarios de carrera tendrán derecho a la promoción profesional, siendo la carrera profesional el conjunto ordenado de oportunidades de ascenso y expectativas de progreso profesional conforme a los principios de igualdad, mérito y capacidad. A tal objeto las Administraciones Públicas promoverán la actualización y perfeccionamiento de la cualificación profesional de sus funcionarios de carrera ${ }^{(9)}$.

Se prevé así mismo en esta Ley un sistema de grados, categorías o escalones de ascenso, fijándose la remuneración a cada uno de ellos. Los ascensos serán consecutivos con carácter general salvo en aquellos supuestos excepcionales en los que se prevea otra posibilidad. Se deberá valorar la trayectoria y actuación profesional, la calidad de los trabajos realizados, los conocimientos adquiridos y el resultado de la evaluación del desempeño. Podrán incluirse asimismo otros méritos y aptitudes por razón de la especificidad de la función desarrollada y la experiencia adquirida.

El desarrollo de los principios generales de la carrera profesional para personal sanitario que desarrolla sus funciones en organizaciones no sanitarias debería confluir con los principios generales para el reconocimiento de desarrollo profesional para el personal sanitario que desarrolla sus funciones en organizaciones sanitarias enunciados en el artículo 38 de la Ley 44/2003, de 21 de noviembre, de ordenación de las profesiones sanitarias ${ }^{(10)}$. 
En segundo lugar, el desarrollo de la medicina evaluadora puede verse encauzado en el contexto legal actual como un área de capacitación específica que dote del mayor rigor científico posible a las actuaciones de los profesionales médicos.

Para ello la Ley de ordenación de profesiones sanitarias establece que el Gobierno, de acuerdo con el procedimiento legalmente establecido, puede establecer Áreas de Capacitación Específica dentro de una o varias Especialidades en Ciencias de la Salud.

El Diploma de Área de Capacitación Específica tiene carácter oficial y validez en todo el territorio del Estado. Se expide por el Ministerio de Sanidad y su posesión será necesaria para utilizar de modo expreso la denominación de especialista con capacitación específica en el área. Podrá ser valorado como mérito para acceder a puestos de trabajo de alta especialización en centros o establecimientos públicos y privados.

\section{Conclusiones}

Como primera conclusión, en el ámbito de los profesionales propios de la seguridad social los médicos funcionarios de carrera tienen el derecho a la promoción profesional a través de un conjunto ordenado de oportunidades de ascenso y expectativas de progreso profesional conforme a los principios de igualdad, mérito y capacidad, lo que implica un sistema de carrera profesional para el colectivo de médicos inspectores adscritos al Instituto Nacional de la Seguridad Social.

Como segunda conclusión, la medicina evaluadora tiene un cuerpo de conocimientos muy amplio, mucho más de lo que se percibe por sectores que no practican esta rama de la medicina, que se evidencia en la celebración de reiterados masters y cursos de actualización en patologías y en herramientas específicas para ejercer esta rama no asistencial de la medicina con garantías para el paciente, para el propio médico, para la institución de la seguridad social y en definitiva, para el conjunto de la sociedad. Todo ello pone en evidencia la necesidad de una formación adicional específica para que, desde cualquier especialidad de la medicina, se pueda asegurar la correcta evaluación médica de las limitaciones orgánicas y funcionales de sus pacientes, enfocadas a un ámbito laboral y de prestaciones de seguridad social.

La implementación, ya urgente, de estas dos medidas, en opinión de los autores, tendría un reflejo directo en el servicio público que presta la Seguridad Social a la ciudadanía, que demanda una mejora continuada de la praxis evaluadora médica y, por tanto, dotaría de mayor rigor a la Administración de la Seguridad Social que, en esta materia, precisa apoyarse en conocimientos especializados y basados en la evidencia científica objetivada.

\section{Bibliografía}

1. Regal Ramos R. El médico inspector de la Seguridad Social. Med Segur Trab 2014;60 (Sup 1):12-16.

2. Arancón Viguera A. Teoría y Práctica de la Medicina Evaluadora. $1^{a}$ ed. Madrid, España: Temis. Fundación Mapfre Medicina; 2004.

3. Constitución Española. Boletín Oficial del Estado, núm. 311, de 29/12/1978.

4. Real Decreto Legislativo $8 / 2015$, de 30 de octubre, por el que se aprueba el texto refundido de la Ley General de la Seguridad Social. Boletín Oficial del Estado, núm. 261, de 31/10/2015.

5. Real Decreto 1300/1995, de 21 de julio, por el que se desarrolla, en materia de incapacidades laborales del sistema de la Seguridad Social, la Ley 42/1994, de 30 de diciembre, de medidas fiscales, administrativas y de orden social. Boletín Oficial del Estado, núm. 198, de 19/08/1995.

6. Real Decreto 2583/1996, de 13 de diciembre, de estructura orgánica y funciones del Instituto Nacional de la Seguridad Social y de modificación parcial de la Tesorería General de la Seguridad Social. Boletín Oficial del Estado, núm. 3, de 3/10/1997. 
7. Sánchez-Galán L, Rodríguez-Ortiz de Salazar B, Herreros-Portolés G. La medicina evaluadora como área de capacitación específica. Med Segur Trab. 2004;50(194):5-7.

8. Sánchez-Galán L, Olalla-García T. Deber o derecho: la carrera profesional de los Médicos Evaluadores de la Seguridad Social. Medicina general [revista electrónica] 2007 [Consultado el 15-10-2020] 098:538543. Disponible en: http://semg.info/mgyf/medicinageneral/revista_98/pdf/538-543.pdf

9. Real Decreto Legislativo 5/2015, de 30 de octubre, por el que se aprueba el texto refundido de la Ley del Estatuto Básico del Empleado Público. Boletín Oficial del Estado, núm. 261, de 31/10/2015.

10. Ley $44 / 2003$, de 21 de noviembre, de ordenación de las profesiones sanitarias. Boletín Oficial del Estado, núm. 280, de 22/11/2003. 